\title{
Análise das alterações radiográficas pulmonares durante a terapêutica da paracoccidioidomicose*
}

\author{
Review of lung radiographic findings during treatment of patients with chronic paracoccidioidomycosis
}

Cristian Saievicz de Moraes $^{1}$, Flávio Queiroz-Telles ${ }^{2}$, Edson Marchiori ${ }^{3}$, Dante Luiz Escuissato ${ }^{4}$

Resumo Objetivo: Foram analisadas as alterações radiográficas pulmonares em pacientes com paracoccidioidomicose crônica de modo evolutivo, verificando-se as diferenças entre os tratados com um novo agente antifúngico triazólico, o voriconazol, em relação aos que utilizaram a atual droga de escolha para o tratamento, o itraconazol. Materiais e Métodos: Realizou-se estudo comparativo, randomizado, com avaliação das radiografias do tórax obtidas antes, durante e após o tratamento de 39 pacientes, divididos em dois grupos: um recebendo o voriconazol e o outro, itraconazol. A avaliação das radiografias teve como parâmetro uma adaptação do método já estabelecido para a análise de outra doença pulmonar difusa, o esquema ILO (International Labour Office) de classificação das pneumoconioses, classificando-se as lesões em pequenas ou grandes opacidades. Resultados: Os resultados obtidos coincidiram com as descrições prévias existentes em relação à apresentação das alterações radiográficas pulmonares, e a evolução destas ao longo do tratamento foi semelhante nos dois grupos. As pequenas opacidades regrediram parcialmente, ao passo que as grandes opacidades mostraram tendência à regressão completa. Conclusão: 0 uso da adaptação do modelo ILO de classificação das pneumoconioses revelou-se útil para o acompanhamento dos pacientes com paracoccidioidomicose crônica ao longo do tratamento, do ponto de vista radiográfico.

Unitermos: Paracoccidioidomicose; Tórax; Radiografia; Esquema ILO; Voriconazol.

Abstract Objective: Lung radiographic findings were reviewed to evaluate the differences in the progression of findings along the follow-up of patients with chronic paracoccidioidomycosis treated with a novel second-generation triazole antifungal agent (voriconazole) as compared with patients treated with the drug of choice for treatment of such disease (itraconazole). Materials and Methods: A comparative, randomized study involved the review of chest radiographic images acquired before, during and after treatment of 39 patients divided into two groups: one receiving voriconazole and the other receiving itraconazole. The parameters adopted to describe and to measure lesions present on the radiographic images were based on an adaptation of the method used in cases of another diffuse pulmonary disease, the ILO (International Labour Office) classification of pneumoconioses, dividing the lesions into small and large opacities. Results: The results coincided with previous descriptions regarding lung radiographic findings, and the progression of such findings along the treatment was similar for both groups. Partial regression was observed in the small opacities while large opacities showed a tendency towards complete regression. Conclusion: The adapted ILO classification demonstrated to be useful in the radiographic follow-up along treatment of patients with chronic paracoccidioidomycosis. Keywords: Paracoccidioidomycosis; Chest; Radiography; ILO classification; Voriconazole.

Moraes CS, Queiroz-Telles F, Marchiori E, Escuissato DL. Análise das alterações radiográficas pulmonares durante a terapêutica da paracoccidioidomicose. Radiol Bras. 2011 Jan/Fev;44(1):20-28.

* Trabalho realizado no Serviço de Radiologia do Departamento de Clínica Médica do Hospital de Clínicas da Universidade Federal do Paraná (UFPR), Curitiba, PR, Brasil.

1. Mestre, Médico Radiologista do Hospital de Clínicas da Universidade Federal do Paraná (UFPR) e da Clínica DAPI, Curitiba, PR, Brasil.

2. Doutor, Professor Adjunto da Disciplina de Infectologia do Departamento de Medicina Interna, Diretor de Corpo Clínico do Hospital de Clínicas da Universidade Federal do Paraná (UFPR), Curitiba, PR, Brasil.

3. Professor Titular de Radiologia da Universidade Federal Fluminense (UFF), Niterói, RJ, Brasil.

4. Professor Doutor, Chefe da Disciplina de Radiologia do Departamento de Medicina da Universidade Federal do Paraná (UFPR), Curitiba, PR, Brasil.

Endereço para correspondência: Dr. Cristian Saievicz de Moraes. Rua Lamenha Lins, 635, ap. 801, Rebouças. Curitiba, PR, Brasil, 80250-020. E-mail: saievicz@hotmail.com

Recebido para publicação em 25/6/2010. Aceito, após revisão, em 25/11/2010.

\section{INTRODUÇÃO}

A paracoccidioidomicose ou blastomicose sul-americana (PCM) é uma micose sistêmica cuja evolução natural, caso não haja instituição da terapêutica específica, é para o óbito ${ }^{(\mathbf{1})}$. Restrita ao continente americano, ocorre do México à Argentina. A maior casuística tem sido registrada no Brasil, Argentina, Colômbia e Venezuela ${ }^{(2)}$. No Brasil, a doença é mais frequente nas regiões Sul, Sudeste e Centro-Oeste ${ }^{(3)}$. Acomete predominantemente indivíduos do sexo masculino na faixa etária de 30 a
50 anos, sendo rara abaixo dos 14 anos de idade $^{(4)}$. A PCM é endêmica na população da zona rural, onde se estima que a incidência anual seja de 1 a 3 casos por 100.000 habitantes ${ }^{(\mathbf{5})}$. Das micoses profundas, a PCM é a de maior impacto na saúde pública. Os indivíduos acometidos estão na fase de maior atividade e as sequelas ocasionadas por esta micose são causas comuns de incapacitação para o trabalho. A regressão espontânea das lesões tem sido relatada muito raramente ${ }^{(6)}$.

Ainda que a tomografia computadorizada do tórax com técnica de alta resolução 
(TCAR) seja reconhecidamente superior, tanto para análise do padrão e distribuição das alterações pulmonares quanto para a avaliação de suas complicações (como escavações e desenvolvimento de fibrose), as radiografias simples do tórax continuam sendo o exame com a melhor relação custobenefício para a detecção e seguimento dos pacientes $^{(7)}$.

Vários antifúngicos sistêmicos podem ser empregados no tratamento da PCM, como o itraconazol, um derivado triazólico de primeira geração. Recentemente, novos derivados triazólicos foram desenvolvidos. Entre estes, o voriconazol caracteriza-se por apresentar amplo espectro antifúngico, incluindo agentes de micoses invasivas e endêmicas, como o Paracoccidioides brasiliensis, agente etiológico da $\mathrm{PCM}^{(\mathbf{8})}$.

Os objetivos desta análise foram:

1 - Revisar as alterações radiográficas pulmonares que ocorrem na PCM crônica de modo evolutivo, antes, durante e após o tratamento, com relação aos seus padrões, distribuição e profusão, utilizando-se critérios definidos, objetivos e reprodutíveis, através da adaptação e simplificação do método já estabelecido para a análise de outra doença pulmonar difusa - o esquema ILO (International Labour Office) de classificação de radiografias de tórax de pacientes com pneumoconioses ${ }^{(\boldsymbol{9})}$.

2 - Verificar se existem diferenças no resultado do tratamento com um novo agente antifúngico triazólico, o voriconazol, e a atual droga de escolha para o tratamento da PCM crônica, o itraconazol, do ponto de vista da evolução radiográfica das lesões pulmonares.

\section{MATERIAIS E MÉTODOS}

A análise radiológica fez parte de uma avaliação global, comparativa e randomizada, envolvendo a terapêutica da PCM crônica, realizada prospectiva e simultaneamente em três centros de referência brasileiros. Foram analisadas as radiografias do tórax, na incidência posteroanterior, de 39 pacientes consecutivos, portadores de PCM crônica com comprometimento pulmonar, obtidas antes, durante e após o tratamento antifúngico. Os pacientes foram divididos em dois grupos: um recebendo como tratamento o voriconazol e outro o itraconazol, randomizados, respectivamente, na proporção de dois para um.

Foram incluídos indivíduos de ambos os sexos, maiores de 18 anos de idade. Pacientes do sexo feminino não poderiam engravidar durante o estudo, pelo fato de serem os derivados triazólicos contraindicados na gestação. O diagnóstico foi microbiologicamente documentado pelo achado do $P$. brasiliensis em diversos tecidos e/ou material biológico, por meio de exame micológico direto, histopatologia e/ou cultura. $\mathrm{O}$ estudo foi conduzido segundo as normas de boas práticas de pesquisa clínica e todos os participantes assinaram termo de consentimento informado.

Cinco casos foram excluídos da análise final: quatro por não terem completado o tempo mínimo de tratamento e um por necessitar de tratamento prolongado, devido a comprometimento concomitante do sistema nervoso central.

\section{Definição dos grupos de estudo}

Cada paciente foi identificado por um código numérico e, após processo de aleatorização, todos passaram a receber uma das duas drogas. Dos 34 pacientes que completaram o estudo, 21 foram tratados com voriconazol e 13 utilizaram o itraconazol (droga controle). Caso a resposta clínica global ao fim de seis meses não fosse satisfatória, a critério do pesquisador, o tratamento poderia ser prolongado por até mais seis meses.

\section{Análise radiográfica - a adaptação e simplificação do sistema ILO}

Os exames radiográficos foram realizados no momento do diagnóstico e randomização dos pacientes (basal), um mês, dois meses e três meses após o início do tratamento, ao término do tratamento (final) e dois meses após o término do tratamento (exame de seguimento). A análise dos exames foi realizada de maneira independente por dois radiologistas, resolvendo-se de maneira consensual eventuais opiniões discordantes. Para que a avaliação se tornasse o mais reprodutível e objetiva possível, empregou-se uma adaptação do esquema ILO de classificação das pneumoconioses de $1980^{(9)}$, em que cinco são os parâmetros de análise: 1) pequenas opacidades arredondadas; 2) pequenas opacida- des irregulares; 3) profusão das pequenas opacidades; 4) grandes opacidades; 5) extensão das lesões.

Pequenas opacidades arredondadas são definidas como imagens nodulares bem delimitadas com diâmetro inferior a $10 \mathrm{~mm}$, como exemplificado nas radiografias de tórax pré-tratamento de dois pacientes do estudo (Figuras 1 e 2).

Pequenas opacidades irregulares referem-se às lesões descritas como lineares, reticulares ou reticulonodulares. Neste estudo, foram classificadas como pequenas opacidades as imagens lineares, reticulares ou reticulonodulares cujo menor diâmetro era inferior a $10 \mathrm{~mm}$ (Figura 2).

A profusão (número de pequenas opacidades, arredondadas ou irregulares, por unidade de área ou por zona pulmonar) das alterações foi avaliada conforme quatro categorias: categoria $\mathbf{0}$, pequenas opacidades ausentes; categoria I, pequenas opacidades definitivamente presentes, porém, em pequeno número, não chegando a comprometer a visualização das imagens vasculares normais; categoria II, numerosas pequenas opacidades, obscurecendo parcialmente as imagens vasculares normais; e categoria III, pequenas opacidades bastante numerosas, não sendo possível distinguir as imagens vasculares normais. Para fins de análises estatísticas, empregou-se uma escala de valores ordinais variando de 0 a 3, conforme maior ou menor profusão das lesões (Tabela 1).

O termo grandes opacidades foi usado para lesões com padrão alveolar maiores que 10 mm (Figura 3), subdividindo-se em quatro categorias: categoria $\mathbf{O}$, ausência de grandes opacidades; categoria $\mathbf{A}$, opacidade com maior diâmetro acima de $1 \mathrm{~cm}$ e no máximo $5 \mathrm{~cm}$ ou várias opacidades com diâmetro individual superior a $1 \mathrm{~cm}$ e cuja soma dos seus maiores diâmetros não ultrapassa $5 \mathrm{~cm}$; a categoria $\mathbf{B}$ compreende uma ou mais opacidades maiores e mais numerosas que as da categoria $\mathbf{A}$, cuja área combinada não exceda a área da zona pulmonar superior direita; já a categoria $\mathbf{C}$ consiste de uma ou mais opacidades, cuja área combinada é maior do que a área da zona pulmonar superior direita. Utilizou-se também uma escala de valores numerais ordinais variando de 0 a 3 para graduação das grandes opacidades (Tabela 2). 


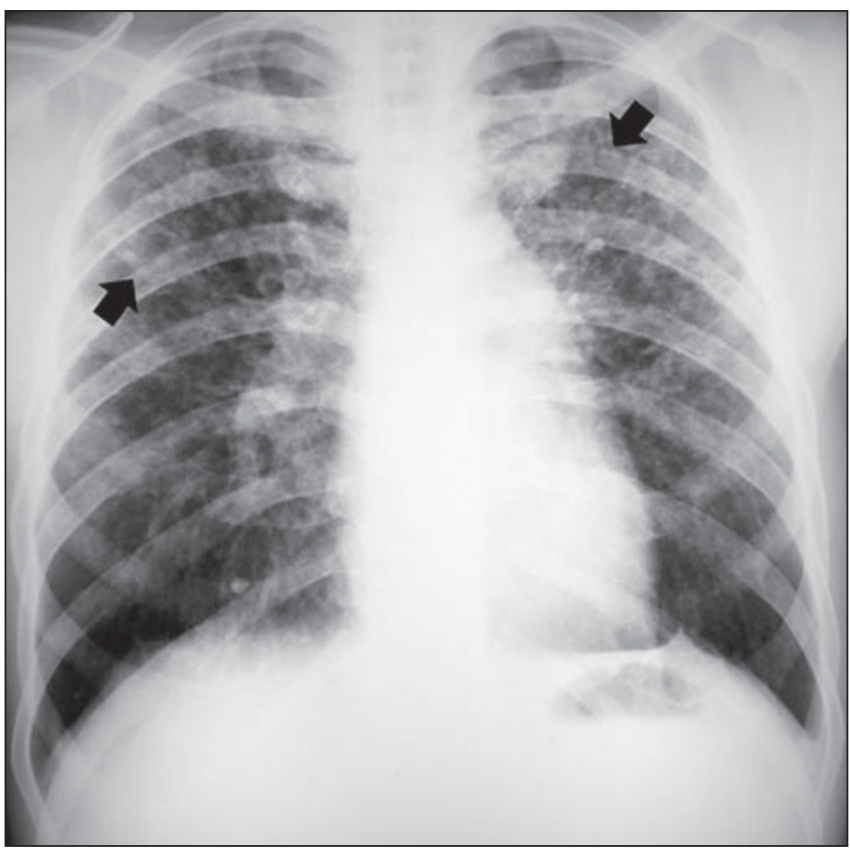

Figura 1. Pequenas opacidades arredondadas (setas).

Tabela 1 Categorização da profusão das pequenas opacidades.

\begin{tabular}{ccc}
\hline Categoria & $\begin{array}{c}\text { Visualização das imagens } \\
\text { vasculares }\end{array}$ & $\begin{array}{c}\text { Valor ordinal } \\
\text { atribuído }\end{array}$ \\
\hline 0 & Sim & 0 \\
I & Sim & 1 \\
II & Parcial & 2 \\
III & Não & 3 \\
\hline
\end{tabular}

Tabela 2 Categorização da extensão das grandes opacidades.

\begin{tabular}{ccc}
\hline Categoria & Dimensões & $\begin{array}{c}\text { Valor ordinal } \\
\text { atribuído }\end{array}$ \\
\hline 0 & $*$ & 0 \\
A & $>1 \mathrm{~cm} \mathrm{e}<5 \mathrm{~cm}$ & 1 \\
B & $>5 \mathrm{~cm} \mathrm{e}<$ que a área da zona $1^{\dagger}$ & 2 \\
C & $>$ que a área da zona 1 & 3 \\
\hline
\end{tabular}

* Não se aplica; ${ }^{\dagger}$ Zona pulmonar superior direita.

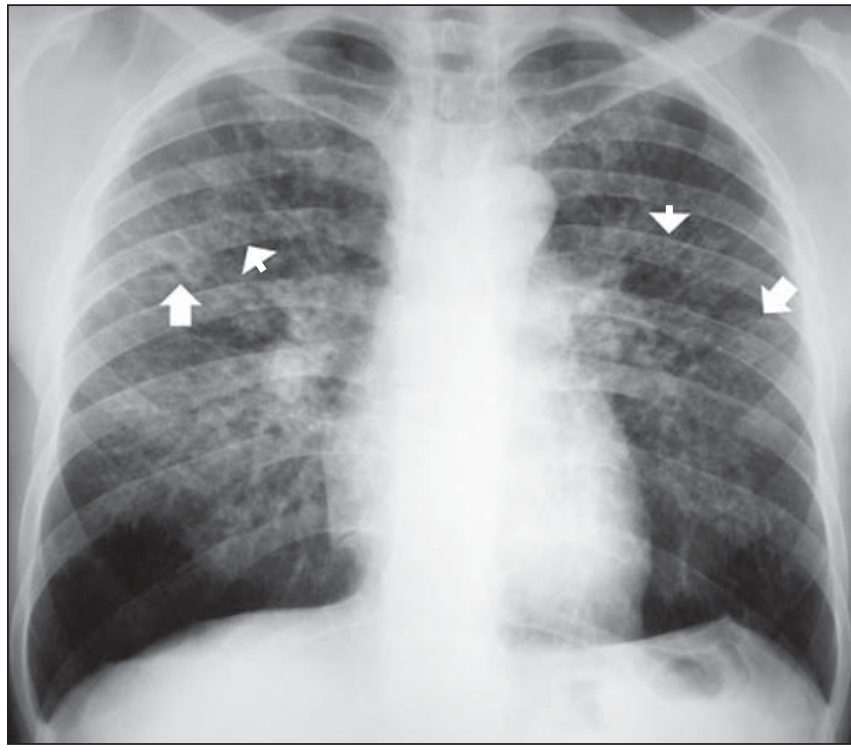

Figura 2. Pequenas opacidades irregulares (setas) e pequenas opacidades arredondadas (cabeças de setas).

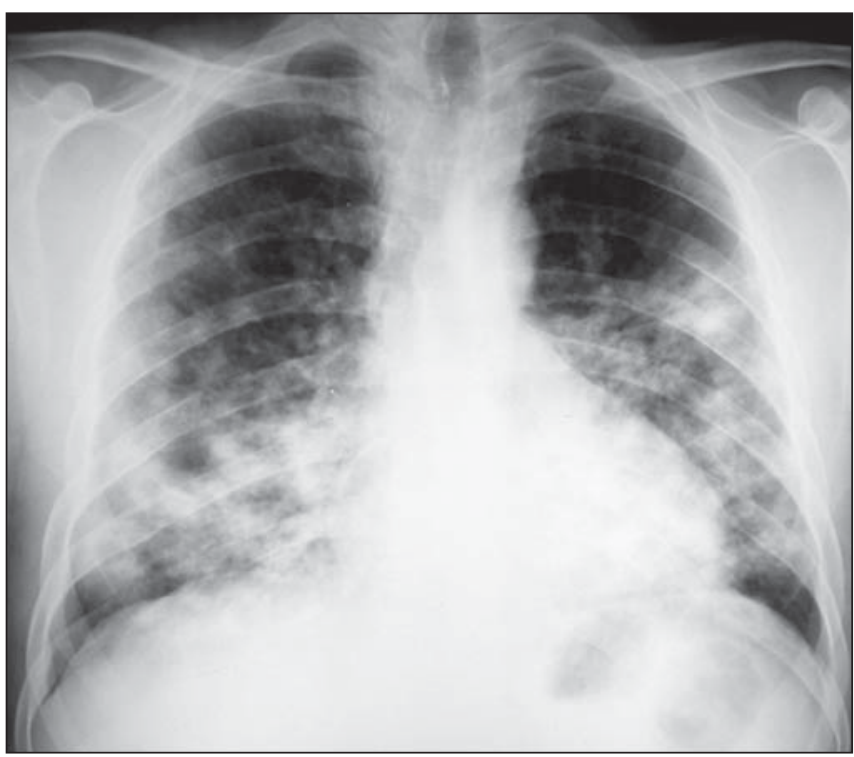

Figura 3. Grandes opacidades nos dois terços inferiores dos pulmões.
Para avaliar a extensão e localização das lesões, cada pulmão foi repartido em três zonas - superior, média e inferior - por linhas horizontais que dividem a distância vertical do ápice pulmonar até a cúpula diafragmática em três partes iguais. As zonas pulmonares foram denominadas do seguinte modo: superior direita $=$ zona 1 , média direita $=$ zona 2, inferior direita $=$ zona 3 , superior esquerda $=$ zona 4 , média esquerda $=$ zona 5 e inferior esquerda $=$ zona 6 (Figura 4).

Em cada uma das seis zonas, nos seis diferentes tempos, foi atribuído um valor ordinal variando entre o mínimo de zero $(0)$ e o máximo de seis (6), conforme a profusão das pequenas opacidades e a presença/extensão das grandes opacidades. Deste modo, se, por exemplo, a zonas pulmonares inferiores de determinado paciente (Fi- gura 5) apresentassem pequenas opacidades em profusão tal que obscurecessem completamente as imagens vasculares pulmonares normais, e grandes opacidades cuja extensão total excedesse $5 \mathrm{~cm}$, porém não ultrapassando a área da zona pulmonar superior direita, o valor ordinal atribuído para cada um destes campos seria igual a 5 (categoria $\mathbf{I I I}=3+$ categoria $\mathbf{B}=2$ ) . Ainda na Figura 5 observam-se nas zonas pulmo- 


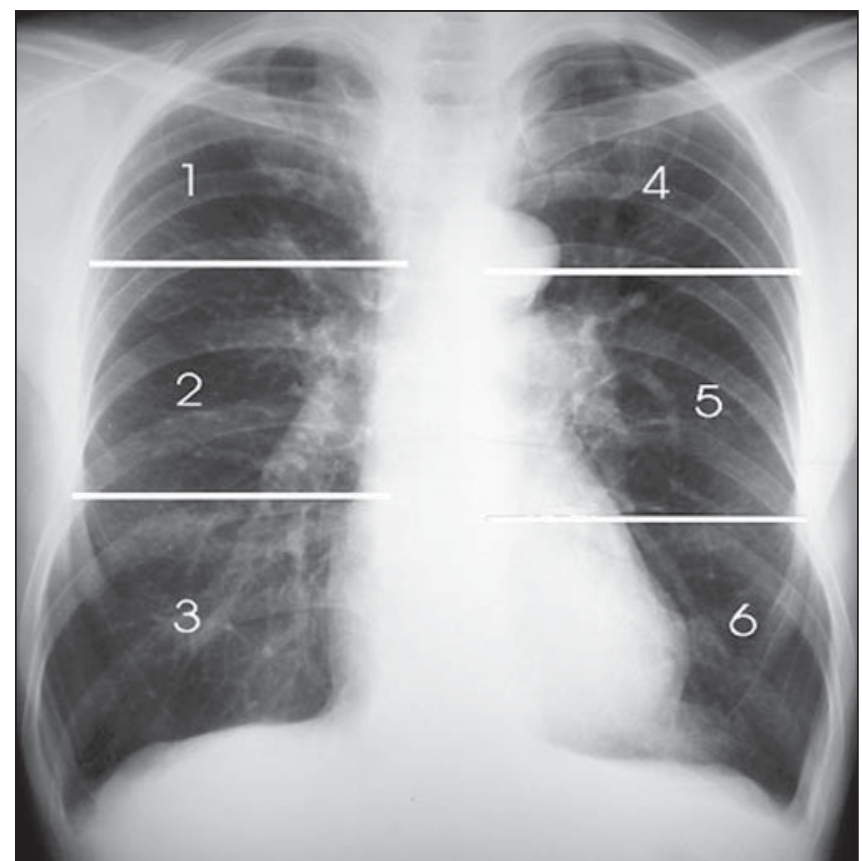

Figura 4. Divisão dos pulmões em seis zonas.

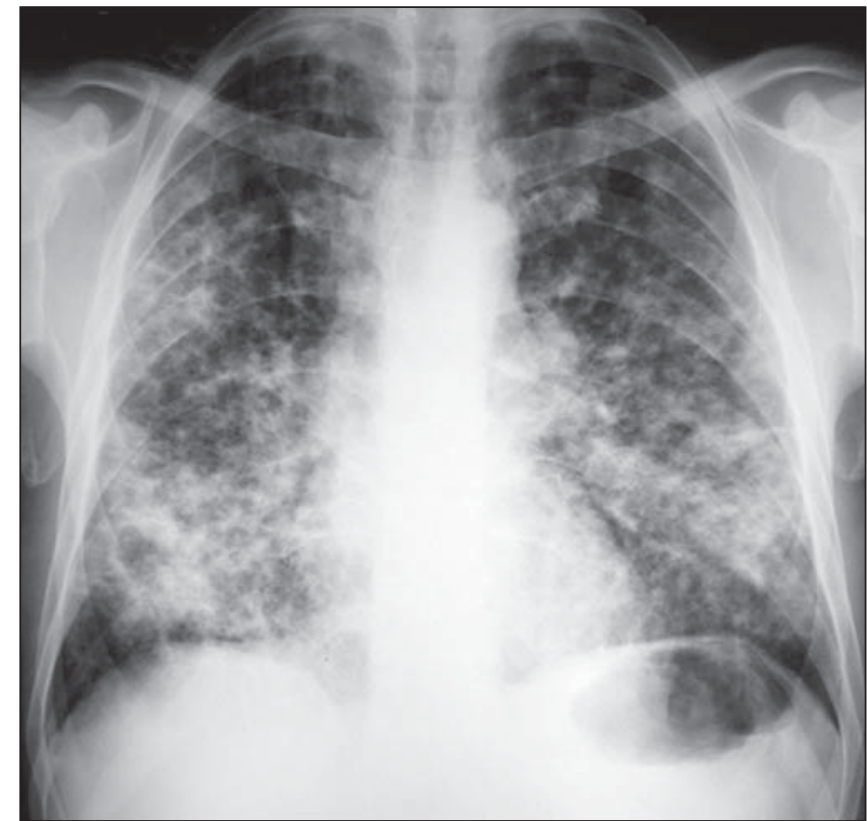

Figura 5. Lesões pulmonares difusas, bilaterais e simétricas, predominando nas zonas médias e inferiores. nares médias somente pequenas opacidades, que impedem parcialmente a visualização das imagens vasculares normais, atribuindo-se para cada uma destas zonas valor igual a 2 (categoria $\mathbf{I I}=2$ ).

Em cada um dos seis diferentes tempos somaram-se os valores das seis zonas pulmonares, obtendo-se um valor em escala ordinal (situado entre o mínimo de 0 e o máximo de 36) que quantifica as alterações pulmonares presentes na radiografia de tórax em posteroanterior de cada paciente, em determinado momento do estudo.

\section{Análise estatística}

Nas comparações entre amostras independentes (diferentes tratamentos), testouse a hipótese de resultados iguais. Para isso, adotou-se o teste não paramétrico de Mann-Whitney.

Na comparação entre amostras pareadas (diferentes tempos de análise das radiogra- fias de um mesmo paciente), testou-se a hipótese de resultados iguais em dois momentos, adotando-se o teste não paramétrico de Wilcoxon.

Em todos os casos, observou-se o nível de significância de 5\% $(p<0,05)$ e a opção pelos testes não paramétricos foi em função dos resultados não terem sido avaliados diretamente, mas por meio de uma escala $^{(10)}$.

As variáveis foram definidas de acordo com: o momento de realização do exame radiográfico, as divisões dos pulmões e a lesão pulmonar.

\section{RESULTADOS}

A grande maioria dos casos era do sexo masculino $(94,1 \%)$ e suas demais características estão resumidas na Tabela 3 .

A Tabela 4 demonstra, para os dois grupos de pacientes, as medianas e as varia- ções (valores máximos e mínimos) das somas dos valores obtidos em cada zona pulmonar, em cada momento de análise.

As notas representativas da profusão das lesões pulmonares obtidas em cada uma das seis zonas, nos momentos basal e ao final, variaram de 0 a 5 , e os valores medianos encontram-se na Tabela 5 .

\section{Comparação entre os tratamentos}

De acordo com controles de cura clínica, laboratorial e radiológica, foram necessários seis meses para o tratamento dos pacientes dos dois grupos, o que está em concordância com os achados de outros estudos $^{(\mathbf{1 1})}$. Do ponto de vista da análise das imagens radiográficas, a suficiência dos seis meses de tratamento ficou evidente pelo fato de que não houve modificações significativas das radiografias no final do tratamento e no seguimento dois meses após (Tabela 4).

Tabela 3 Composição dos dois diferentes grupos de tratamento.

\begin{tabular}{|c|c|c|c|c|c|c|c|c|}
\hline \multirow[b]{3}{*}{ Medicação recebida } & \multirow{2}{*}{\multicolumn{2}{|c|}{ Sexo }} & \multicolumn{2}{|c|}{ Idade } & \multirow{2}{*}{\multicolumn{2}{|c|}{ Procedência }} & \multicolumn{2}{|c|}{ Tempo de tratamento } \\
\hline & & & \multirow{2}{*}{$\begin{array}{c}\text { Variação } \\
\text { (anos) }\end{array}$} & \multirow{2}{*}{$\begin{array}{l}\text { Média } \\
\text { (anos) }\end{array}$} & & & \multirow{2}{*}{$\begin{array}{l}\text { Seis } \\
\text { meses }\end{array}$} & \multirow{2}{*}{$\begin{array}{l}\text { Superior } \\
\text { a seis meses }\end{array}$} \\
\hline & Masculino & Feminino & & & Zona rural & Zona urbana & & \\
\hline Voriconazol (21 pacientes) & $20(95,2 \%)$ & $1(4,8 \%)$ & $31-66$ & 48,1 & $19(90,5 \%)$ & $2(9,5 \%)$ & $19(90,48 \%)$ & $2(9,52 \%)$ \\
\hline Itraconazol (13 pacientes) & $12(92,35)$ & $1(7,7 \%)$ & $40-66$ & 44,7 & $9(69,2 \%)$ & $4(30,8 \%)$ & $11(84,62 \%)$ & $2(15,38 \%)$ \\
\hline Total (34 pacientes) & $32(94,1 \%)$ & $2(5,9 \%)$ & $31-66$ & 46 & $28(82,4 \%)$ & $6(17,6 \%)$ & 30 & 4 \\
\hline
\end{tabular}


Tabela 4 Medianas das somas dos valores obtidos em cada zona pulmonar nos diferentes momentos de análise.

\begin{tabular}{cccccc}
\hline & Basal & Um mês & Dois meses & Três meses & Final \\
\hline $\begin{array}{c}\text { Voriconazol } \\
\text { Mediana (variação) } \\
\begin{array}{c}\text { Itraconazol } \\
\text { Mediana (variação) }\end{array}\end{array}$ & $9(0-21)$ & $8(0-23)$ & $7(0-20)$ & $4(0-19)$ & $4(0-15)$ \\
\hline
\end{tabular}

Tabela 5 Medianas dos valores obtidos em cada zona pulmonar nos momentos basal e final.

\begin{tabular}{|c|c|c|c|c|c|c|c|c|c|c|c|c|}
\hline & \multicolumn{2}{|c|}{ Zona 1} & \multicolumn{2}{|c|}{ Zona 2} & \multicolumn{2}{|c|}{ Zona 3} & \multicolumn{2}{|c|}{ Zona 4} & \multicolumn{2}{|c|}{ Zona 5} & \multicolumn{2}{|c|}{ Zona 6} \\
\hline & Basal & Final & Basal & Final & Basal & Final & Basal & Final & Basal & Final & Basal & Final \\
\hline Voriconazol $(n=21)$ & 1 & 0 & 2 & 1 & 2 & 1 & 1 & 0 & 2 & 1 & 1 & 0 \\
\hline Itraconazol $(n=14)$ & 2 & 0 & 3 & 0 & 1 & 0 & 0 & 0 & 0 & 0 & 1 & 0 \\
\hline
\end{tabular}

Os resultados de média \pm um desviopadrão validaram a hipótese de resultados iguais nos dois tratamentos $(p>0,05) \mathrm{e}$ demonstraram que no início do estudo os dois grupos eram homogêneos em relação às alterações radiográficas, considerandose tanto o pulmão como um todo como as zonas pulmonares individualmente $(p>$ $0,05)$.

Ao término do tratamento, a melhora radiográfica em cada zona pulmonar também foi semelhante $(p>0,05)$.

\section{Comparação entre os momentos de análise}

Nos dois grupos houve regressão gradual das alterações radiográficas pulmonares ao longo de todo o tratamento $(p<$ $0,05)$, como exemplificam as Figuras 6 e 7.

\section{Comparação entre as zonas pulmonares}

Ao início do tratamento observou-se, nos dois grupos, diferença significativa da profusão das lesões entre as zonas superiores e médias $(p<0,05)$, não havendo dife- rença significativa entre a profusão de lesões em campos médios e inferiores.

No momento basal notou-se, no grupo tratado com itraconazol, diferença significativa da profusão das lesões em zonas pulmonares superiores versus inferiores ( $p<0,05)$, e pôde-se inferir que há uma distribuição simétrica das lesões, sem diferença significativa de resultados nas comparações entre zonas superiores, médias ou inferiores direitas e esquerdas $(p>0,05)$. Tal distribuição, bilateral e simétrica, com

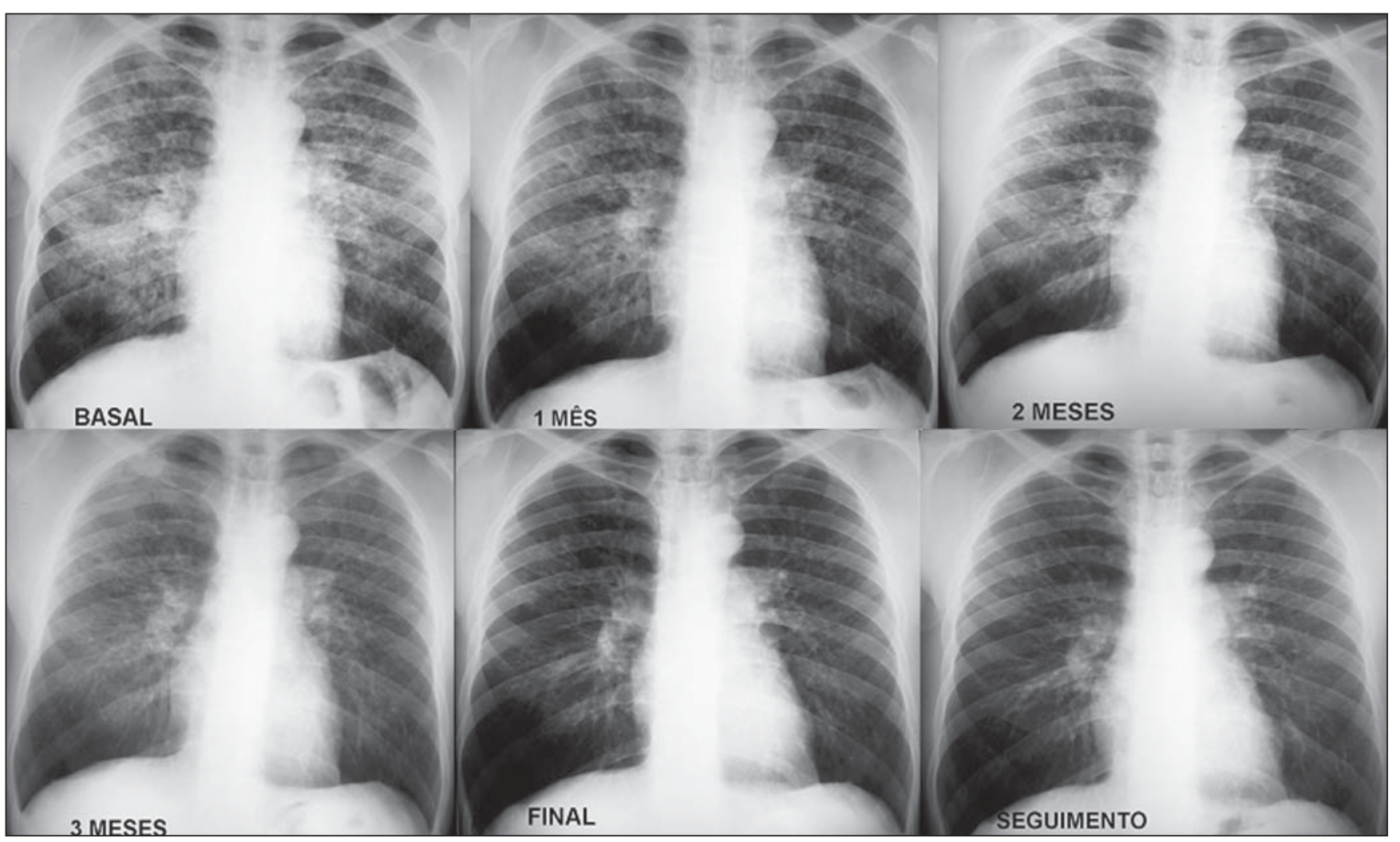

Figura 6. Melhora contínua e gradual das alterações radiográficas pulmonares em paciente tratado com voriconazol. 


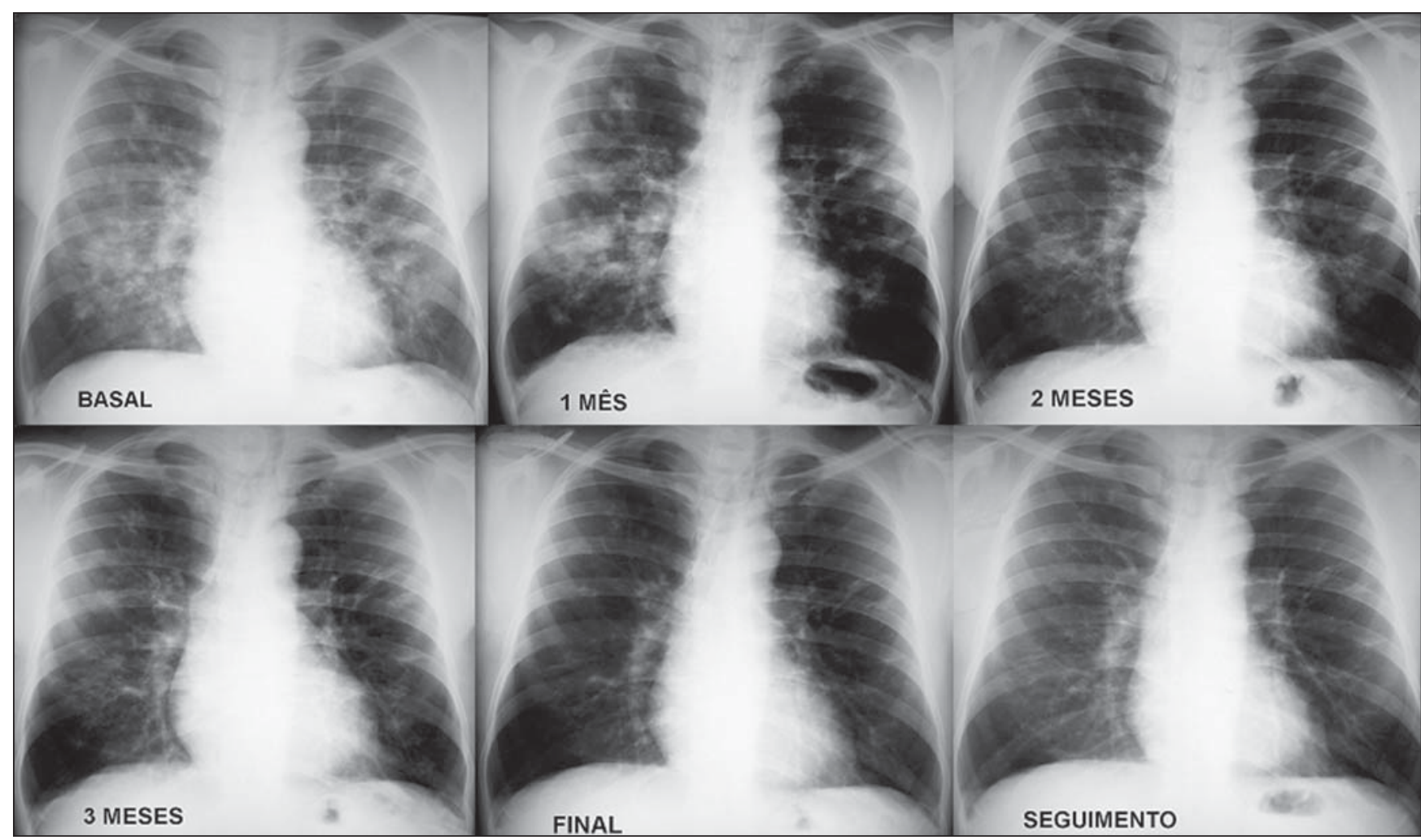

Figura 7. Melhora contínua e gradual das alterações radiográficas pulmonares em paciente tratado com itraconazol.

predomínio pelas zonas pulmonares médias e inferiores, pode ser observada nas $\mathrm{Fi}-$ guras 3 e 5 .

Observou-se, ainda, tendência de que as diferenças entre a profusão de lesões nas diversas zonas pulmonares permaneçam após o tratamento.

\section{Evolução das pequenas e grandes opacidades}

No momento basal, $67,6 \%$ dos pacientes apresentavam como alterações nas radiografias do tórax, exclusivamente, pequenas opacidades $(69,2 \%$ do pacientes tratados com itraconazol e $66,7 \%$ dos que receberam voriconazol), 29,4\% apresentavam tanto pequenas como grandes opacidades (30,8\% do grupo do itraconazol e $28,6 \%$ dos que receberam voriconazol), e em apenas um caso $(2,9 \%)$ não foram evidenciadas alterações radiográficas.

Ao fim do tratamento houve regressão parcial das pequenas opacidades em 27 pacientes $(79,4 \%)$, e em 6 pacientes $(17,6 \%)$ as lesões permaneceram estáveis desde o início da análise. Já as grandes opacidades sofreram regressão completa em nove dos dez casos que as apresentavam no tempo basal, seis do grupo tratado com voriconazol e quatro do grupo do itraconazol (resolução das grandes opacidades em $90 \%$ dos

Tabela 6 Regressão da profusão das pequenas opacidades e da extensão das grandes opacidades com o tratamento.

\begin{tabular}{lcccccc}
\hline & \multicolumn{2}{c}{ Pequenas opacidades } & & \multicolumn{2}{c}{ Grandes opacidades* } \\
\cline { 2 - 3 } & \multicolumn{2}{c}{ Basal } & Final & & Basal & Final \\
\hline Voriconazol - mediana (variação) & $9(0-18)$ & $4(0-14)$ & & $2(1-5)$ & $0(0-2)$ \\
Itraconazol - mediana (variação) & $11(1-18)$ & $4(0-8)$ & & $4(2-6)$ & $0(0-0)$ \\
\hline
\end{tabular}

* Os valores da extensão das grandes opacidades referem-se apenas aos pacientes que as apresentavam no tempo basal.

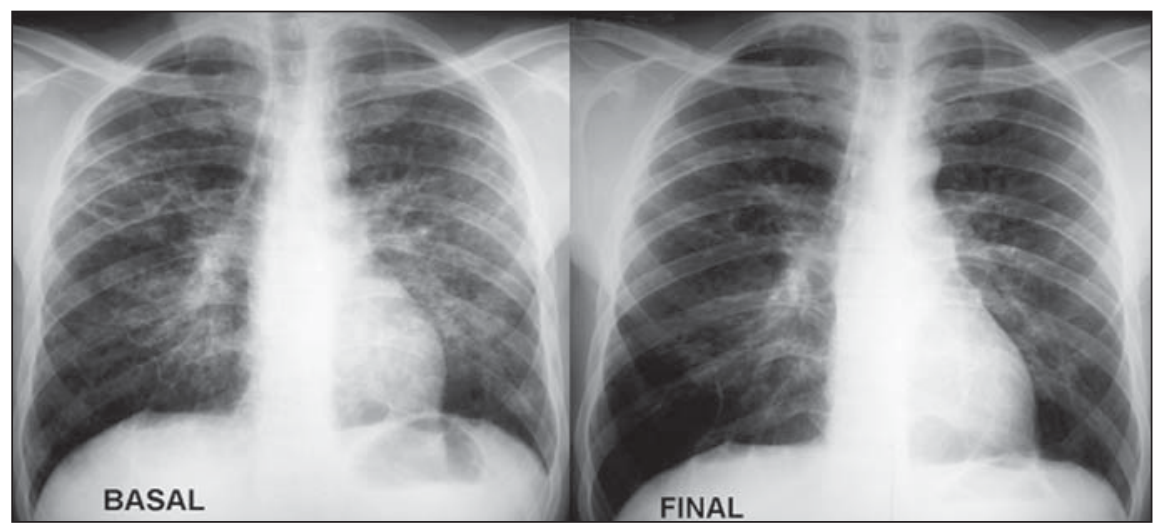

Figura 8. Regressão das pequenas opacidades com o tratamento, restando estrias irregulares nos campos pulmonares médios, de provável natureza cicatricial. 


\section{DISCUSSÃO}

A nomenclatura utilizada nas classificações radiológicas propostas para as alterações pulmonares encontradas na PCM crônica é muito variável, não havendo consenso ou uma classificação universalmente aceita. As classificações variam de lesões micronodulares, infiltrativas e estriadas ${ }^{(\mathbf{1 2})}$, passando por formas produtivas, produtivo-exsudativas e produtivo-exsudativo-escavadas $^{(\mathbf{1 3 )}}$, até padrões infiltrativo, nodular e fibrótico ${ }^{(14)}$. Dessa forma, para que as alterações pudessem ser qualificadas e quantificadas de maneira objetiva e reprodutível, optou-se por dividi-las, conforme classificação adaptada do esquema ILO, em pequenas e grandes opacidades.

A distribuição bilateral e simétrica das lesões, predominando nas zonas médias e inferiores, confirmou os achados de estudos prévios $^{(12,15,16)}$. Outros autores também encontraram lesões bilaterais, porém com predomínio franco pelos campos pulmonares médios ou regiões peri-hilares ${ }^{(17-19)}$.

Reafirmou-se a pequena frequência do padrão conhecido como em "asa de borboleta", caracterizado por opacidades alveolares bilaterais, nos campos pulmonares médios. Nenhum dos 34 pacientes apresentou, exclusivamente, opacidades alveolares nos campos pulmonares médios. Entretanto, padrão semelhante, correspondendo a grandes opacidades nos campos médios e também em alguns outros campos, foi observado em dois pacientes $(5,88 \%)$. Cruz et al. chamaram esta forma de pseudopneumônica, encontrando-a em $0,5 \%$ dos $\operatorname{casos}^{(20)}$. Este padrão foi encontrado em $10 \%$ dos casos acompanhados por Martins et al. ${ }^{(21)}$, em $16,6 \%$ por Valle et al. ${ }^{(22)} \mathrm{e}$ em 2,7\% por Machado Filho e Miranda ${ }^{(\mathbf{1 2})}$.

Já no trabalho de Funari et al., em que o método de avaliação das alterações pulmonares foi a TCAR, constatou-se distribuição bilateral, simétrica, nos diversos campos pulmonares, havendo tendência a poupar as regiões anteriores das zonas inferiores $^{(7)}$, o que, de certa forma, coincide com o sinal do "Y invertido" nas radiografias de tórax em perfil, descrito por Quagliato Jr., em que há acometimento preferencial da língula, lobo médio e regiões posteriores dos lobos superiores e inferiores ${ }^{(23)}$. Estudo mais recente também mos- tra distribuição das alterações em porções periféricas e posteriores dos pulmões, com discreta predominância pelas zonas pulmonares médias ${ }^{(24)}$. Esta tendência de que as porções anteriores das zonas pulmonares inferiores sejam poupadas não foi avaliada no presente estudo, pois os dados obtidos basearam-se nas radiografias obtidas na incidência posteroanterior.

Os padrões radiográficos básicos já descritos são: lesões nodulares, lesões reticulares e condensações ou opacidades alveolares, com as suas possíveis combinações.

No presente trabalho, os nódulos foram classificados como pequenas opacidades arredondadas, com diâmetro inferior a 10 $\mathrm{mm}$, e não foram avaliadas as diferentes dimensões que esses nódulos apresentavam entre si. Já as opacidades nodulares maiores foram classificadas como grandes opacidades.

O nódulo já foi definido como a lesão radiológica fundamental na PCM com envolvimento pulmonar, ocorrendo com variadas dimensões, desde miliares até macronodulares, em um mesmo paciente ${ }^{(25)}$. Cruz et al. confirmaram a variabilidade das dimensões dos nódulos e referiram-se às formas macronodulares ou pseudotumorais como blastomicomas ${ }^{(\mathbf{2 0})}$. Segundo Valle et al., enquanto os nódulos teriam entre $4 \mathrm{~mm}$ e $5 \mathrm{~mm}$ de diâmetro, os micronódulos estariam compreendidos entre $1 \mathrm{~mm} \mathrm{e} 2 \mathrm{~mm}^{(22)}$. Opinião discordante foi proposta em estudo anterior a estes, em que foram descritas apenas as formas micronodulares ${ }^{(12)}$. Com a utilização da TCAR, confirmou-se a variabilidade da dimensão dos nódulos, muitas vezes com contornos irregulares ${ }^{(7,24)}$.

Há dificuldade para separar as formas reticulares puras das reticulonodulares nas radiografias simples do tórax. Assim, optou-se por classificá-las também como pequenas opacidades, cujos menores diâmetros não excedem $10 \mathrm{~mm}$. As classificações já propostas não definem se estas alterações pertencem ao grupo das lesões infiltrativas passíveis de resolução com o tratamento ou se já corresponderiam a alterações intersticiais fibrogênicas residuais. Neste sentido, as manifestações clínicas poderiam auxiliar, já que os pacientes com infiltrados alveolointersticiais não fibrogênicos geralmente apresentam lesões em mucosas, além de odinofagia e/ou disfagia, ao passo que os pacientes com alterações pulmonares fibrogênicas apresentam-se com lesões na derme e dispneia ${ }^{(26)}$.

$\mathrm{Na}$ PCM crônica, as lesões cicatriciais não ocasionam grandes deformidades retráteis como as que ocorrem na tuberculo$\mathrm{se}^{(17)}$, e para definir a sua presença faz-se necessária análise evolutiva das radiografias ao longo do tratamento.

As condensações alveolares e opacidades, nodulares ou não, maiores que $10 \mathrm{~mm}$, foram classificadas como grandes opacidades. Já foi sugerido que as opacidades alveolares resultariam da coalescência dos infiltrados reticulares ou reticulonodulares $^{(18)}$. O substrato anatomopatológico das opacidades alveolares seria uma reação pneumônica, em que há alveolite aguda com múltiplos fungos entre as células inflamatórias ou no interior de histiócitos ${ }^{(27)}$. Em cerca de $10 \%$ dos casos tal alveolite aguda pode circundar áreas de inflamação intersticial, resultando, à TCAR, no padrão denominado "sinal do halo invertido"(28).

No presente estudo, as pequenas opacidades corresponderiam ao padrão intersticial e as grandes, ao padrão alveolar. Nos dois grupos de tratamento, cerca de dois terços dos pacientes apresentaram o padrão intersticial, somente com pequenas opacidades nas radiografias do tórax, e aproximadamente um terço, o padrão misto com alterações pertencentes tanto ao grupo das pequenas como ao das grandes opacidades. Não foram observados casos contendo exclusivamente grandes opacidades (padrão alveolar puro), e em apenas um dos pacientes os estudos radiográficos do tórax foram sempre considerados como normais. Talvez a TCAR evidenciasse alterações neste paciente com radiografias normais, embora trabalho anterior utilizando a TCAR tenha encontrado uma pequena porcentagem (7\%) de pacientes com PCM crônica sem anormalidades pulmonares parenquimato$\operatorname{sas}^{(7)}$. A maioria das pesquisas anteriores também demonstrou predomínio dos padrões intersticial ou misto, em relação ao padrão alveolar ${ }^{(12,22)}$.

Em concordância com a maioria das séries em que não foram utilizados exames tomográficos computadorizados ou planigráficos, não foram caracterizadas, com segurança, lesões escavadas. As escavações ocorrem em focos prévios de condensação 
e a adequada detecção e análise delas exige estudos tomográficos computadorizados ou exames de planigrafia linear ${ }^{(\mathbf{2 0})}$. No trabalho de Funari et al. com 41 pacientes, em que o método de análise foi a TCAR, lesões escavadas cujos diâmetros variavam entre $1 \mathrm{~cm} \mathrm{e} \mathrm{4,8} \mathrm{cm} \mathrm{foram} \mathrm{encontradas} \mathrm{em} 17,1 \%$ dos $\operatorname{casos}^{(7)}$. Outros estudos encontraram escavações em uma frequência $36 \% \%^{(\mathbf{2 9}, 30)}$ e $42,9 \%{ }^{(25)}$. Na TCAR também são descritas escavações com paredes irregulares e com septos internos $^{(\mathbf{3 1})}$.

Não foram observados atelectasias, envolvimento pleural, linfonodomegalias ou calcificações. A ausência de calcificações é outro dado que auxilia no diagnóstico diferencial com a tuberculose, enfatizando-se, inclusive, que se procurem doenças associadas, como tuberculose ou histoplasmose, caso haja calcificações ${ }^{(\mathbf{1 6})}$. Ressalta-se ainda mais um ponto de diferenciação com a tuberculose: a raridade da atelectasia lobar, pela tendência de não ocorrerem lesões de natureza obstrutiva nos brônquios fontes e lobares na PCM crônica ${ }^{(\mathbf{1 2})}$.

Observou-se regressão contínua e gradual da profusão das alterações radiográficas em 27 dos 34 pacientes. Em um caso não foi possível evidenciar alterações e seis casos apresentaram lesões estáveis nos diversos momentos de análise. O caso com radiografias do tórax consideradas como normais já havia recebido tratamento com sulfas anteriormente e apresentava alterações em estudo radiográfico prévio, realizado cinco anos antes. Dois dos casos com lesões estáveis também já haviam sido tratados para PCM, irregularmente. Os quatro casos restantes com alterações imutáveis não haviam sido tratados, porém, antes de iniciarem o tratamento atual, já vinham apresentando sinais e sintomas da doença por períodos que variavam de quatro meses a dois anos. O longo tempo de sintomas e o tratamento antifúngico prévios tornam viável a possibilidade de que já houvesse alterações cicatriciais no momento basal de análise.

A maioria dos estudos anteriores encontrou melhora mais acentuada das manifestações radiográficas no terceiro mês de tratamento ${ }^{(32,33)}$

As pequenas opacidades sofreram regressão parcial, enquanto as grandes só não regrediram completamente em um dos dez casos que as apresentavam no momento basal. Assim, pode-se sugerir que a resolução das áreas de consolidação alveolar e dos macronódulos seria um indicativo de resposta adequada ao tratamento utilizado.

Ao término do tratamento, as pequenas opacidades predominavam nos campos pulmonares médios e inferiores, permanecendo com distribuição bilateral e simétrica, associadas a aumento de áreas hipertransparentes periféricas, que poderiam corresponder a enfisema ${ }^{(\mathbf{1 5})}$, bolhas ${ }^{(\mathbf{3 4})}$ ou até mesmo áreas de aprisionamento de ar devido ao comprometimento de pequenas vias aéreas.

Dessa forma, resultaram como conclusões:

1. Confirmaram-se os achados de análises prévias existentes na literatura com relação aos padrões, distribuição e profusão das alterações radiográficas pulmonares em pacientes com PCM crônica.

2. Não houve diferenças estatisticamente significativas na evolução radiográfica torácica da PCM crônica em pacientes tratados com voriconazol e itraconazol.

3. O uso de uma adaptação do modelo ILO de classificação das pneumoconioses mostrou-se útil para a avaliação objetiva da evolução das alterações radiográficas pulmonares difusas presentes em pacientes com PCM crônica, ao longo do tratamento.

\section{Agradecimentos}

Os autores agradecem ao Ambulatório de Doenças Infecciosas e Parasitárias do Hospital de Clínicas da Universidade Federal do Paraná e aos seus pacientes, por disponibilizarem os estudos radiográficos torácicos utilizados neste trabalho.

\section{REFERÊNCIAS}

1. Montenegro MR, Franco M. Pathology. In: Franco M, Lacaz CS, Restrepo-Moreno A, et al., editors. Paracoccidioidomycosis. Boca Raton: CRC Press; 1994. p. 131-50.

2. Bethlem NM, Lemle A, Bethlem E, et al. Paracoccidioidomycosis. Semin Respir Med. 1991; 12:81-97

3. Wanke B, Londero AT. Epidemiology and paracoccidioidomycosis infection. In: Franco M, Lacaz CS, Restrepo-Moreno A, et al., editors. Paracoccidioidomycosis. Boca Raton: CRC Press; 1999. p. 109-20.

4. Londero AT, Ramos CD. Paracoccidioidomicose. Estudo clínico e micológico de 260 casos observados no interior do Estado do Rio Grande do Sul. J Pneumol. 1990;16:129-32.

5. Restrepo A, Greer DL. Paracoccidioidomycosis.
In: DiSalvo AF, editor. Occupational mycoses. Philadelphia: Lea \& Febiger; 1983. p. 43-64.

6. Melo IS, Londero AT. Spontaneously resolving pulmonary lesions in paracoccidioidomycosis. Case report and review. Mycopathologia. 1983; 82:57-9.

7. Funari M, Kavakama J, Shikanai-Yasuda MA, et al. Chronic pulmonary paracoccidioidomycosis (South American blastomycosis): high resolution CT findings in 41 patients. AJR Am J Roentgenol. 1999;173:59-64.

8. Espinel-Ingroff A. In vitro activity of the new triazole voriconazole (UK-109,496) against opportunistic filamentous and dimorphic fungi and common and emerging yeast pathogens. J Clin Microbiol. 1998;36:198-202.

9. International Labour Office. Guidelines for the use of ILO International Classification of Radiographs of Pneumoconiosis. Occupational Safety and Health Series No. 22. Geneva: International Labour Office; 1980. p. 1-48.

10. Glantz SA. Primer of biostatistics. 5th ed. San Francisco: McGraw-Hill; 2002.

11. Naranjo MS, Trujillo M, Munera MI, et al. Treatment of paracoccidioidomycosis with itraconazole. J Med Vet Mycol. 1990;28:67-76.

12. Machado Filho J, Miranda JL. Considerações relativas à blastomicose sul-americana. Da participação pulmonar entre 338 casos consecutivos. O Hospital. 1960;58:23-42.

13. Passos Filho MCR. Blastomicose sul-americana. Comentários em torno de 83 casos de localização pulmonar - classificação radiológica. O Hospital. 1966;70:127-52.

14. Gutiérrez F, Silva M, Peláez F, et al. The radiological appearances of pulmonary paracoccidioidomycosis and the effects of ketoconazole therapy. J Pneumol. 1985;11:1-12.

15. Rodríguez C, Rincón NL, Troconis-García G Contribution to the study of paracoccidioidomycosis brasiliensis in Venezuela. Considerations on 62 cases studied with special reference to respiratory localizations. Mycopathologia. 1961; 15:115-38

16. Hernandez HS. Clinical and radiological aspects of paracoccidioidomycosis. Proceedings of the First Pan American Symposium on Paracoccidioidomycosis; 1971 Oct; Medellín, Colombia.

17. Gonçalves AP, Bardy C. Aspectos clínicos e radiológicos da blastomicose brasileira pulmonar. O Hospital. 1946;30:213-43.

18. Gutiérrez F. Radiological follow-up in five cases of paracoccidioidomycosis. Proceedings of the First Pan American Symposium on Paracoccidioidomycosis; 1971 Oct; Medellín, Colombia.

19. Magalhães A. Paracoccidioidomicose (blastomicose sul-americana). Aspectos radiológicos. Rev Hosp Clin Fac Med São Paulo. 1980;35:147-53.

20. Cruz MFA, Santos Filho RA, Cardoso RC, et al. Aspectos radiográficos intratorácicos da paracoccidioidomicose. Revisão de 170 casos. Radiol Bras. 1989;22:169-77.

21. Martins S, Gerhardt Filho G, Monteiro DJ. Aspectos clínicos e radiológicos da paracoccidioidomicose. J Bras Med. 1984;46:71-9.

22. Valle ACF, Guimarães RR, Lopes DJ, et al. Aspectos radiológicos torácicos na paracoccidioidomicose. Rev Inst Med Trop São Paulo. 1992;34: 107-15. 
23. Quagliato Jr R. Considerações a respeito da paracoccidioidomicose e sua correlação com níveis séricos de alfa-1-antitripsina [dissertação de mestrado]. Campinas: Universidade Estadual de Campinas; 1980.

24. Souza ASS Jr, Gasparetto EL, Davaus T, et al. High-resolution CT findings of 77 patients with untreated pulmonary paracoccidioidomycosis AJR Am J Roentgenol. 2006;187:1248-52.

25. Bardy C. Sinais radiológicos pulmonares da blastomicose sul-americana. J Bras Med. 1962;6 484-8.

26. Restrepo A, Tobón AM, Agudelo CA, et al. Coexistence of integumentary lesions and lung $\mathrm{X}$-ray abnormalities in patients with paracoccidioidomycosis (PCM). Am J Trop Med Hyg. 2008;79. 159-63.
27. Tuder RM, el Ibrahim R, Godoy CE, et al. Pathology of the human pulmonary paracoccidioidomycosis. Mycopathologia. 1985;92:179-88.

28. Gasparetto EL, Escuissato DL, Davaus T, et al. Reversed halo sign in pulmonary paracoccidioidomycosis. AJR Am J Roentgenol. 2005;184:19324.

29. Kauer CL. Paracoccidioidomicose: tomografia computadorizada de alta resolução das alterações Pleuro-pulmonares [tese de doutorado]. Porto Alegre: Universidade Federal do Rio Grande do Sul; 2000.

30. Muniz MAS, Marchiori E, Magnago M, et al. Paracoccidioidomicose pulmonar: aspectos na tomografia computadorizada de alta resolução. Radiol Bras. 2002;35:147-54.
31. Marchiori E, Moraes HP, Muniz MAS, et al. Paracoccidioidomicose: correlação da tomografia computadorizada de alta resolução com a anatomopatologia. Radiol Bras. 2000;33:333-40.

32. Restrepo A, Gómez I, Robledo J, et al. Itraconazole in the treatment of paracoccidioidomycosis: a preliminary report. Rev Infect Dis. 1987;9 Suppl 1:S51-6.

33. Del Negro G. Ketoconazole in paracoccidioidomycosis. A long-term therapy study with prolonged follow-up. Rev Inst Med Trop São Paulo. 1982;24:27-39.

34. Restrepo A, Gómez I, Cano LE, et al. Treatment of paracoccidioidomycosis with ketoconazole: a three-year experience. Am J Med. 1983;74:4852 